\title{
XXXV. A method of determining the number of signals which can be made by the modern telegraphs
}

\section{Charles Blackburn Esq. A.B.}

To cite this article: Charles Blackburn Esq. A.B. (1834) XXXV. A method of determining the number of signals which can be made by the modern telegraphs, Philosophical Magazine Series 3, 5:28, 241-244, DOI: 10.1080/14786443408648455

To link to this article: http://dx.doi.org/10.1080/14786443408648455

曲 Published online: 01 Jun 2009.

Submit your article to this journal $[\pi$

Џll Article views: 3

Q View related articles $\llbracket$ 


\title{
LONDON AND EDINBURGH
}

\section{PHILOSOPHICAT، MAGAZINE}

\author{
A N D \\ JOURNAL OF SCIENCE. \\ [THIRD SERIES.] \\ $O C T O B E R \quad 1834$.
}

XXXV. A Method of determining the Number of Signals which can be made by the Modern Telegraphs. By ChARLes Blackburn, Esq., A.B.

To the Editors of the Philosophical Magazine and Journal. Gentlemen,

A LTHOUGH the French telegraph or semaphore has $A$ been in use for a considerable period, I am not aware that any general rule for determining the number of signals that can be made by these instruments has been given to the public. The following investigation, therefore, may not be unacceptable to such of your readers as are interested in that mode of communication. Its object is to furnish a rule for determining the number of distinct signals which can be made by any semaphore, whatever be the number of arms or indicators, or whatever be the number of positions of each arm.

In the Cyclopxdia of Rees, the number of signals which the semaphores of the line of communication between Paris and Landau were capable of making, is stated to be 823,543 , which is no less than $1,274,608$ fewer than the real number, an error not arising from the press, but from the principle of computation. The following method, besides giving the true number of signals, has the advantage of being reducible to an expression of remarkable simplicity.

Problem.-To find the number of signals which can be made by any semaphore having any given number of arms, and each arm taking any given number of positions.

Third Series. Vol. 5. No. 28. Oct. 1834. 2 I 
Let $\mathrm{AB}$ be an upright pole or staff, carrying any number of arms $\mathrm{FC}, \mathrm{DG}, \mathrm{HE}$, \&c., moveable in a vertical plane about the centres $F, D, E$, \&c., and capable of being placed in any number of positions. This will represent the modern telegraph or semaphore.

Let the number of centres F, D, E, \&c. be denoted by $c$, and the number of positions of each arm by $p$. Then, since the number of signals that can be made by one arm must be equal to the number of positions of that arm, the number of signals which can be made with one arm $=p$, and the number of signals on the whole, using one arm at a time, will be $c p$.

Again, since each of the signals which can

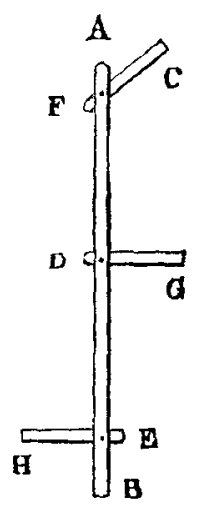
be made by any one arm, can be repeated with each of the signals of any other arm, it follows that the number which can be made by any two arms together $=p^{2}$. And since the number of combinations in $c$ things, taken two and two together, $=\frac{c \cdot \overline{c-1}}{1.2}$, it follows that the whole number of signals, using two arms at once, $=\frac{c \cdot \overline{c-1}}{1.2} \cdot p^{2}$.

In like manner it may be shown that the number of signals which can be made using three arms at once $=\frac{c \cdot \overline{c-1} \cdot \overline{c-2}}{1 \cdot 2 \cdot 3} p^{8}$; and that when all the arms are used at once, the number will be $\frac{c \cdot \overline{c-1} \cdot \overline{c-2} \ldots \ldots . . \overline{c-(c-1)}}{1.2 .3 \ldots \ldots \ldots \ldots \ldots \ldots} p^{\circ}$, the index of $p$ being always $=$ number of arms used at once. The whole number of signals, therefore, which the telegraph is capable of making will be

$$
\begin{aligned}
& +\frac{c \cdot \overline{c-1}}{1.2} \cdot p^{2} \\
& +\frac{c \cdot \overline{c-1} \cdot \overline{c-2}}{1.2 \cdot 3} \cdot p^{8} \\
& +\frac{c \cdot \overline{c-1} \cdot \overline{c-2} \ldots \ldots \cdot \frac{8 c}{c-(c-1)}}{1 \cdot 2 \cdot 3 \ldots \ldots \ldots . c} \cdot p^{c}
\end{aligned}
$$

But if the binomial $1+p$ be raised to the cth power, it will be found to coincide exactly with the sum of the preceding 
terms, with the exception of unity, by which it exceeds it. The expression $(1+p)^{c}-1$, therefore, will accurately represent the whole number of signals.

Example.-In the Admiralty semaphore, the number of centres or arms $=2$, and the number of positions of each arm $=6$. Hence, by the theorem, the number of signals will be $(6+1)^{2}-1=7^{2}-1=48$.

Example 2.-If the number of centres be three, as in the preceding sketch, and the number of positions of each arm be six, the number of signals will be $(6+1)^{3}-1=7^{3}-1=342$.

Example 3.-In the year 1796, a line of telegraphs was established between Paris and Landau, each of which had seven arms, and each arm seven positions. It is required to find the number of signals.

By the theorem, the signals $=(7+1)^{7}-1=8^{7}-1=$ $2,097,151$.

Corollary.-From the preceding investigation it appears that, for any semaphore having $c$ centres, and each arm $p$ positions, the number of signals which can be made, using one arm at once, will be represented by the second term of the binomial $1+p$ raised to the $c$ th power; the number of signals using two arms at once, by the third term of the same quantity, \&c.; and the number of signals, using all the arms at once, by the $(c+1)$ th term.

Thus, in the Admiralty semaphore,

The signals using one arm at once $=c p=2 \times 6=12$

The signals using two arms at once $=\frac{c \cdot \overline{c-1}}{1.2} p^{2}=1 \times 6^{8}=36$

Total number of signals, as before, $=\overline{48}$

In the Paris semaphore before mentioned, the signals using one arm at once $\quad \ldots \quad \ldots \quad \ldots \ldots=c p=7 \times 7=49$ two arms at once $\quad \ldots \quad \ldots=\frac{c \cdot \overline{c-1}}{1.2} p^{2}=21 \times 49=1029$ three arms at once $\ldots \quad \ldots=\frac{c \cdot \overline{c-1} \cdot \overline{c-2}}{1 \cdot 2 \cdot 3} p^{3}=35 \times 343=12005$ four arms $\quad \ldots \quad \ldots=\frac{c \cdot \overline{c-1} \cdot c \overline{-2} \cdot c \overline{-3}}{1 \cdot 2 \cdot 3 \cdot 4} p^{4}=35 \times 2401=84035$ five arms $\ldots=\frac{c \cdot \overline{c-1} \cdot \overline{c-2} \cdot \overline{c-3} \cdot \overline{c-4}}{1 \cdot 2 \cdot 3 \cdot 4 \cdot 5} p^{5}=21 \times 16807=352947$ six arms $=\frac{c \cdot \overline{c-1} \cdot \overline{c-2} \cdot \overline{c-3} \cdot \overline{c-4} \cdot \overline{c-5}}{1 \cdot 2 \cdot 3 \cdot 4 \cdot 5 \cdot 6} p^{6}=7 \times 117649=823543$ seven $=\frac{c \cdot \overline{c-1} \cdot \overline{c-2} \cdot \overline{c-3} \cdot \overline{c-4} \cdot \overline{c-5} \cdot \overline{c-6}}{1 \cdot 2 \cdot 3 \cdot 4 \cdot 5 \cdot 6 \cdot 7} p^{7}=1 \times 823543=823543$ Total number of signals, as before, $=\overline{2,097,151}$ 2 I 2 
The preceding investigation supposes one arm only on the same centre, as in the modern semaphores; but as they may be made with several arms on the same centre, it may be proper on some future occasion to give the method of finding the signals for such as have any given number of arms on each centre, any number of centres, and any number of positions of each arm; an investigation a little more complicated than the preceding, but, like it, capable of reduction to an expression of great simplicity.

If, in any semaphore, instead of one arm on each centre, we suppose as many arms on each centre as each arm has positions, the number of signals will be $2^{a c}-1$.

Thus, if the Admiralty semaphore had six arms on each centre instead of one, the number of signals would be $2^{2 \times 6}-1$ $=4095$ instead of 48 as at present.

It is indifferent in the application of the preceding theorem in what order the centres are placed, but they should be in the same vertical plane, and the plane perpendicular to the spectator's line of vision.

Kensington-square, June 3, 1834.

XXXVI. Remarlis on Mr. Beke's Papers on the Gopherwood, and the former Extension of the Persian Gulf. By W. G. Carter, Esq.

\section{To the Editors of the Philosophical Magazine and Journal. Gentlemen,}

IN Mr. Beke's observations in your April Number, on my paper in that for March, that gentleman represents me as stating, that society previously to the Deluge " existed in a state of infancy as regards its culture and knowledge," and " that he apprehends the evidence we possess on the subject ought to lead us to a very different conclusion." Mr. Beke has here mistaken my meaning. I have not expressed any opinion respecting the general culture and knowledge of mankind at that period. My remark was confined to their navigation only. Of all persons, Noah and his family were the least likely to have been ignorant of any art in the building of vessels and boats which was possessed by the antediluvian world. Whatever knowledge they had of the subject, we may infer that they conveyed to their posterity. Yet have we no reason to suppose, from the history of any country, that the arts of ship-building and navigation had made any considerable progress for many ages after the Flood. How, indeed, were they to have been acquired? Navigation is cultivated by an early, very much by any people, in seeking those sup- 